\title{
Hyperbolic Space Forms and Orbifold Compactification in M-Theory
}

\author{
Andrey A. Bytsenko \\ Depto. de Física, Univ. Estadual de Londrina, Paraná, Brazil \\ E-mail: abyts@uel.br
}

Maria Emília X. Guimarães

Depto. de Matemática, Univ. de Brasília, DF, Brazil

E-mail: marg@unb.br

\section{José Abdalla Helayël-Neto*}

Centro Brasileiro de Pesquisas Físicas, Rio de Janeiro, Brazil

E-mail: helayelecbpf.br

We analyze solutions of supergravity and string theories which involve real hyperbolic spaces. Examples of string compactifications are given in terms of hyperbolic coset spaces of finite volume $\Gamma \backslash \mathbb{H}^{N}$, where $\Gamma$ is a discrete group of isometries of $\mathbb{H}^{N}$. We describe finite flux and the tensor kernel associated with hyperbolic spaces. The special case of an arithmetic geometry of $\Gamma=S L(2, \mathbb{Z}+i \mathbb{Z}) /\{ \pm I d\}$, where $I d$ is the identity matrix, is analyzed. We discuss supersymmetry surviving for supergravity solutions involving real hyperbolic space factors, string-supergravity correspondence and holography principle for a class of conformal field theories.

Fourth International Winter Conference on Mathematical Methods in Physics 09 - 13 August 2004

Centro Brasileiro de Pesquisas Fisicas (CBPF/MCT), Rio de Janeiro, Brazil

\footnotetext{
* Speaker.
} 


\section{Introduction}

In supergravity and string theories the de Sitter, anti de Sitter spaces and $N$-spheres $\mathbb{S}^{N}$ play important role. These spaces as well as $N$-dimensional real hyperbolic spaces $\mathbb{H}^{N}$ naturally arise as the near-horizon solutions of black brane geometries. Spheres and the anti de Sitter spaces, as supergravity solutions, have been extensively investigated (see, for example, [1]). As for the de Sitter spaces, not much calculations have been done for such solutions. The reason relies on the fact that the de Sitter space breaks supersymmetry and is not suitable to describe universes with zero cosmological constant.

The $N$-dimensional real hyperbolic space can be represent as the symmetric space $X=G / K$, where $G=S O_{1}(N, 1)$ and $K=S O(N)$ is a maximal compact subgroup of $G$. Hyperbolic space forms admit Killing spinors [2, 3, 4, 5] but they have infinite volume, and do not seem useful for describing internal spaces in string compactifications. Let us regard $\Gamma$ as a discrete subgroup of $G$ acting isometrically on $X$, and take $X_{\Gamma}$ to be quotient space by that action: $X_{\Gamma}=\Gamma \backslash G / K$. The question of interest is whether the space $X_{\Gamma}$ admits Killing spinors and preserves supersymmetry. Comments on this question for some examples of finite volume hyperbolic spaces can be found in [6]. There is a large class of new string regular compactifications (except possible orbifold points) involving real hyperbolic spaces or their coset spaces with very small $\alpha^{\prime}$ string corrections. Among the possible applications of these solutions, the definition of new $\mathcal{N}=0$ conformal field theories in four dimensions via holography [6] (see also [7, 8]), and new models for dimensional reduction in non-compact spaces.

In this paper we discuss solutions of the eleven-dimensional supergravity which can be presented by means of direct product of spaces containing real hyperbolic space forms as factors. We derive the Laplace operator on $p$-forms, the trace formula applied to the tensor kernel and spectral functions of compact hyperbolic spaces. We take into account the orbifolding of the discrete group $\Gamma$. Finally we discuss the questions of supersymmetry surviving under the orbifolding, and string-supergravity correspondence in its connection to the holographic principle.

\section{Hyperbolic sectors in eleven-dimensional backgrounds}

In eleven-dimensional supergravity the graviton multiplet contains the graviton $\mathrm{g}_{M N}$, the antisymmetric three-form $A_{M N K}$ and the gravitino $\Psi_{M}(M, N, K, \ldots=0,1, \ldots, 10)$. The bosonic part of the supergravity Lagrangian has the form

$$
\mathcal{L}_{\text {(boson) }}=\frac{1}{2 \kappa_{11}^{2}} \sqrt{\mathrm{g}}\left(R-\frac{1}{2 \cdot 4 !} F_{M N P Q} F^{M N P Q}\right)-\frac{1}{12^{4}} \varepsilon^{M_{1} \ldots M_{11}} A_{M_{1} M_{2} M_{3}} F_{M_{4} \ldots M_{7}} F_{M_{8} \ldots M_{11}} .
$$

A solution to the equations of motions

$$
\begin{aligned}
R_{M N} & =\frac{1}{12}\left(F_{M P Q R} F_{N} P Q R-\frac{1}{12} \mathrm{~g}_{M N} F^{2}\right), \\
\nabla_{M} F^{M N P Q} & =-\frac{1}{1152} \varepsilon^{N P Q R_{1} \ldots R_{8}} F_{R_{1} \ldots R_{4}} F_{R_{5} \ldots R_{8}},
\end{aligned}
$$

is provided by the Freund-Rubin ansatz for the antisymmetric field strength

$$
F_{m n p q}=6 m_{0} \varepsilon_{m n p q} \text { for } m, n, \ldots=7, \ldots, 10 ; \quad F_{M N P Q}=0, \text { otherwise } .
$$


By substituting this ansatz into the field equations (2.2) we get

$$
R_{\mu v}=-6 m_{0}^{2} \mathrm{~g}_{\mu v}, \quad \mu, v=0, \ldots 6, \quad R_{m n}=12 m_{0}^{2} \mathrm{~g}_{m n} .
$$

The requirement of unbroken supersymmetry, i.e., the vanishing of the gravitino transformation

$$
\delta \Psi_{M}=\nabla_{M} \varepsilon-\frac{1}{288}\left(\Gamma_{M}^{P Q R S}-8 \delta_{M}^{P} \Gamma^{Q R S}\right) \varepsilon F_{P Q R S}
$$

for the ansatz (2.4), is equivalent to the existence of $S O(1,6)$ and $S O(4)$ Killing spinors $\theta$ and $\eta$, respectively, which satisfy

$$
\nabla_{\mu} \theta= \pm \frac{1}{2} m_{0} \gamma_{\mu} \theta, \quad \nabla_{m} \eta= \pm m_{0} \gamma_{m} \eta
$$

where $\gamma_{\mu}\left(\gamma_{m}\right)$ are $S O(1,6)(S O(4)) \gamma$-matrices. Eq. (2.5) admits a solution of the form $X^{7} \times Y^{4}$ where $X^{7}$ and $Y^{4}$ are Einstein spaces of negative and positive curvature, respectively. But only those spaces that admit Killing spinors obeying Eq. (2.7) preserve supersymmetry. The integrability conditions of Eq. (2.7) are $W_{\mu v \rho \sigma} \gamma^{\rho \sigma} \theta=0, W_{\text {mnpq }} \gamma^{p q} \eta=0$, where $W_{\mu v \rho \sigma}, W_{\text {mnpq }}$ are the Weyl tensors of $X^{7}, Y^{4}$, respectively. Thus, obvious supersymmetric examples for $Y^{4}$ include the round foursphere $\mathbb{S}^{4}$ and its orbifolds $\Gamma \backslash \mathbb{S}^{4}$, where $\Gamma$ is an appropriate discrete group [9]. For the $X^{7}$ space one can take the anti de Sitter space $A d S_{7}$, which preserves supersymmetry as well, and leads to the $A d S_{7} \times \mathbb{S}^{4}$ vacuum of eleven-dimensional supergravity. There are solutions to Eq. (2.5) involving hyperbolic spaces which are vacua of eleven-dimensional supergravity, and solve Eqs. (2.2), (2.3):

(i) $\quad A d S_{7-N} \times \mathbb{H}^{N} \times \mathbb{S}^{4}, \quad N \geq 2$

(ii) $\quad A d S_{3} \times \mathbb{H}^{2} \times \mathbb{H}^{2} \times \mathbb{S}^{4}$

(iii) $\quad A d S_{2} \times \mathbb{H}^{2} \times \mathbb{H}^{3} \times \mathbb{S}^{4}$

\section{Hyperbolic geometry in type II supergravity}

\subsection{Fluxes on $G / K$}

First we note that a conformal field theory involving the upper half three-space $\mathbb{H}^{3}$ can be constructed as a Wess-Zumino-Witten (WZW) model based on the coset $S L(2, \mathbb{C}) / S U(2)$. In particular, this theory can be combined with the NS5-brane by summing both conformal sigma models. In the time direction one needs to add a linear dilaton in order to saturate the central gauge. Such a construction leads to an NS-NS two-form gauge field with imaginary components. But by S-duality it could be converted into a R-R two-form with imaginary components, which leads to a solution of type IIB* theory. Thus, as a result the conformal model is an exact solution of string theory to all orders in the $\alpha^{\prime}$-expansion. These sigma models can also be constructed directly by brane intersections [6, 10]. To construct solutions with brane charges, we could start with the NS5 brane, with flux on $\mathbb{H}^{3}$, which is a formal analog of the NS five brane solution, in which the three-sphere is replaced by a hyperbolic space. By S-duality, and redefining the RR two-form, this result could be converted into a solution of type IIB* supergravity.

Near horizon solution of the discussing model is a direct product of a flat space with linear dilaton and a $S L(2, \mathbb{C}) / S U(2)$ WZW model. Also the near-horizon geometry of the NS5 brane with 
flux on $\mathbb{H}^{3}$ describes a background $A d S_{3} \times \mathbb{S}^{3} \times \mathbb{H}^{3} \times S^{1}$, with a linear dilaton in the time direction [6]. By U-duality, one can construct also different D-brane solutions with time dependence. In order to have finite flux, the space $\mathbb{H}^{3}$ can be replaced by the finite volume $\Gamma \backslash \mathbb{H}^{3}$ space.

\subsection{Cusp forms}

We begin with the Dirac heat kernel $\operatorname{Tr}\left(\mathfrak{D} e^{-t \mathfrak{D}}\right)$ which could be expanded as a series of orbital integrals associated to the conjugacy classes $[\gamma]$ in $\Gamma$. Each orbital integral, over a necessary semisimple orbit, can be in turn expressed in terms of the noncommutative Fourier transform of the heat kernel, along the tempered unitary dual of $G$, the group of isometries of the symmetric space $G / K$. The results of [11] on the series expansion for the case of compact locally symmetric spaces of higher ranks has been extended to the odd dimensional non-compact spaces with cusps in [12]. More precisely, taking into account the fixed Iwasawa decomposition $G=K A N$, consider a $\Gamma$-cuspidal minimal parabolic subgroup $G_{P}$ of $G$ with the Langlands decomposition $G_{P}=B A N, B$ being the centralizer of $A$ in $K$. Let us define the Dirac operator $\mathfrak{D}$, assuming a spin structure for $\Gamma \backslash \operatorname{Spin}(2 k+1,1) / \operatorname{Spin}(2 k+1))$. The spin bundle $E_{\tau_{s}}$ is the locally homogeneous vector bundle defined by the spin representation $\tau_{s}$ of the maximal compact group $\operatorname{Spin}(2 k+1)$. One can decompose the space of sections of $E_{\tau_{s}}$ into two subspaces, which are given by the half spin representations $\sigma_{ \pm}$of $\operatorname{Spin}(2 k) \subset \operatorname{Spin}(2 k+1)$. Let us consider a family of functions $\mathcal{K}_{\mathbb{t}}$ over $G=\operatorname{Spin}(2 k+1,1)$, which is given by taking the local trace for the integral kernel $\exp \left(-t \mathfrak{D}^{2}\right)$ (or $\left.\mathfrak{D} \exp \left(-t \mathfrak{D}^{2}\right)\right)$. The Selberg trace formula applied to the scalar kernel function $\mathcal{K}_{t}$ holds [12]:

$$
\begin{aligned}
\sum_{\sigma=\sigma_{ \pm}} \sum_{\lambda_{k} \in \sigma_{p}^{ \pm}} \hat{\mathcal{K}}_{t}\left(\sigma, i \lambda_{k}\right) & -\frac{i}{4 \pi} \int_{\mathbb{R}} d s \operatorname{Tr}\left(S_{\Gamma}\left(\sigma_{ \pm},-s\right) \frac{d}{d s} S_{\Gamma}\left(\sigma_{+}, s\right) \pi_{\Gamma}\left(\sigma_{+}, s\right)\left(\mathcal{K}_{t}\right)\right) \\
& =I_{\Gamma}\left(\mathcal{K}_{t}\right)+H_{\Gamma}\left(\mathcal{K}_{t}\right)+U_{\Gamma}\left(\mathcal{K}_{t}\right)
\end{aligned}
$$

where $\sigma_{p}:=\sigma_{p}^{+} \bigcup \sigma_{p}^{-}$gives the point spectrum of $\mathfrak{D}, S_{\Gamma}\left(\sigma_{+}, i \lambda\right)$ is the intertwining operator and $I_{\Gamma}\left(\mathcal{K}_{t}\right), H_{\Gamma}\left(\mathcal{K}_{t}\right), U_{\Gamma}\left(\mathcal{K}_{t}\right)$ are the identity, hyperbolic and unipotent orbital integrals. If $\mathcal{K}_{t}$ is given by $\mathfrak{D} e^{-t \mathfrak{D}^{2}}$, then $I_{\Gamma}\left(\mathcal{K}_{t}\right)=0$ by the Fourier transformation of $\mathcal{L}_{t}$. The analysis of the unipotent orbital integral $U_{\Gamma}\left(\mathcal{L}_{t}\right)$ gives the following result [13, 12]: All of the unipotent terms vanish in the Selberg trace formula applied to the odd kernel function $\mathcal{K}_{t}$ given by $\mathfrak{D} e^{-t \mathfrak{D}^{2}}$. It means that one can obtain spectral invariants in the case of cusps similar to that in the case of smooth compact odd dimensional manifolds.

\section{The arithmetic geometry of $\Gamma=S L(2, \mathbb{Z}+i \mathbb{Z}) /\{ \pm I d\}$}

Let $\tau$ be an irreducible representation of $K$ on a complex vector space $V_{\tau}$, and form the induced homogeneous vector bundle $G \times_{K} V_{\tau}$. Restricting the $G$ action to $\Gamma$ we obtain the quotient bundle $E_{\tau}=\Gamma \backslash\left(G \times_{K} V_{\tau}\right) \longrightarrow X_{\Gamma}=\Gamma \backslash X$ over $X$. The natural Riemannian structure on $X$ (therefore on $X_{\Gamma}$ ) induced by the Killing form (, ) of $G$ gives rise to a connection Laplacian $\mathfrak{L}$ on $E_{\tau}$. If $\Omega_{K}$ denotes the Casimir operator of $K$-that is $\Omega_{K}=-\sum y_{j}^{2}$, for a basis $\left\{y_{j}\right\}$ of the Lie algebra $\mathfrak{k}_{0}$ of $K$, where $\left(y_{j}, y_{\ell}\right)=-\delta_{j \ell}$, then $\tau\left(\Omega_{K}\right)=\lambda_{\tau} \mathbf{1}$ for a suitable scalar $\lambda_{\tau}$. Moreover for the Casimir operator $\Omega$ of $G$, with $\Omega$ operating on smooth sections $\Gamma^{\infty} E_{\tau}$ of $E_{\tau}$ one has $\mathfrak{L}=\Omega-\lambda_{\tau} \mathbf{1}$. For $\lambda \geq 0$ let $\Gamma^{\infty}\left(X_{\Gamma}, E_{\tau}\right)_{\lambda}=\left\{s \in \Gamma^{\infty} E_{\tau} \mid-\mathfrak{L} s=\lambda s\right\}$ be the space of eigensections of $\mathfrak{L}$ corresponding to $\lambda$. 
Here we note that if $X_{\Gamma}$ is compact we can order the spectrum of $-\mathfrak{L}$ by taking $0=\lambda_{0}<\lambda_{1}<$ $\lambda_{2}<\cdots ; \lim _{j \rightarrow \infty} \lambda_{j}=\infty$. We shall specialize $\tau$ to be the representation $\tau^{(p)}$ of $K=S O(N)$ on $\Lambda^{p} \mathbb{C}^{N}$. It will be convenient moreover to work with the normalized Laplacian $\mathfrak{L}=-c(N) \mathfrak{L}$ where $c(N)=2(N-1)=2(2 k-1) . \mathfrak{L}$ has spectrum $\left\{c(N) \lambda_{j}, m_{j}\right\}_{j=0}^{\infty}$ where the multiplicity $m_{j}$ of the eigenvalue $c(N) \lambda_{j}$ is given by $m_{j}=\operatorname{dim} \Gamma^{\infty}\left(X_{\Gamma}, E_{\tau(p)}\right) \lambda_{j}$.

Let us consider group of local isometry associated with a simple three-dimensional complex Lie group. The discrete group can be chosen in the form $\Gamma \subset P S L(2, \mathbb{C}) \equiv S L(2, \mathbb{C}) /\{ \pm I d\}$, where $I d$ is the $2 \times 2$ identity matrix and is an isolated element of $\Gamma$. The group $\Gamma$ acts discontinuously at point $z \in \overline{\mathbb{C}}, \overline{\mathbb{C}}$ being the extended complex plane. We consider a special discrete group $S L(2, \mathbb{Z}+$ $i \mathbb{Z}) /\{ \pm I d\}$, where $\mathbb{Z}$ is the ring of integer numbers. The element $\gamma \in \Gamma$ will be identified with $-\gamma$. The group $\Gamma$ has, within a conjugation, one maximal parabolic subgroup $\Gamma_{\infty}$. Let us consider an arbitrary integral operator with kernel $k\left(z, z^{\prime}\right)$. Invariance of the operator is equivalent to fulfillment of the condition $k\left(\gamma z, \gamma z^{\prime}\right)=k\left(z, z^{\prime}\right)$ for any $z, z^{\prime} \in \mathbb{H}^{3}$ and $\gamma \in P S L(2, \mathbb{C})$. So the kernel of the invariant operator is a function of the geodesic distance between $z$ and $z^{\prime}$. It is convenient to replace such a distance with the fundamental invariant of a pair of points $u\left(z, z^{\prime}\right)=\left|z-z^{\prime}\right|^{2} / y y^{\prime}$, thus $k\left(z, z^{\prime}\right)=k\left(u\left(z, z^{\prime}\right)\right)$. Let $\lambda_{j}$ be the isolated eigenvalues of the self-adjoint extension of the Laplace operator and let us introduce a suitable analytic function $h(r)$ and $r_{j}^{2}=\lambda_{j}-1$. It can be shown that $h(r)$ is related to the quantity $k(u(z, \gamma z))$ by means of the Selberg transform. Let us denote by $g(u)$ the Fourier transform of $h(r)$, namely $g(u)=(2 \pi)^{-1} \int_{\mathbb{R}} d r h(r) \exp (-i r u)$.

Theorem 1. Suppose that $h(r)$ is an even analytic function in the strip $|\Im r|<1+\varepsilon(\varepsilon>0)$, and $h(r)=O\left(1+|r|^{2}\right)^{-2}$. For the special discrete group $S L(2, \mathbb{Z}+i \mathbb{Z}) /\{ \pm I d\}$ the Selberg trace formula holds

$$
\begin{aligned}
\sum_{j} h\left(r_{j}\right) & -\sum_{\substack{\{\gamma\}_{\Gamma}, \gamma \neq I d, \gamma-\text { non }- \text { parabolic }}} \int d \mu(z) k(u(z, \gamma z)) \\
& -\left.\frac{1}{4 \pi} \int_{\mathbb{R}} d r h(r) \frac{d}{d s} \log S(s)\right|_{s=1+i r}+\frac{h(0)}{4}[S(1)-1]-C g(0) \\
& =\operatorname{Vol}(\Gamma \backslash G) \int_{0}^{\infty} \frac{d r r^{2}}{2 \pi^{2}} h(r)-\frac{1}{4 \pi} \int_{\mathbb{R}} d r h(r) \psi(1+i r / 2) .
\end{aligned}
$$

The first term in the right hand site of Eq. (4.1) is the contribution of the identity element, $\operatorname{Vol}(\Gamma \backslash G)$ is the (finite) volume of the fundamental domain with respect to the measure $d \mu, \psi(s)$ is the logarithmic derivative of the Euler $\Gamma$-function, and $C$ is a computable real constant [14, 15, 16]. The function $S(s)$ is given by a generalised Dirichlet series, convergent for $\Re s>1, S(s)=$ $\pi^{1 / 2} \Gamma(s-1 / 2)[\Gamma(s)]^{-1} \sum_{c \neq 0} \sum_{0 \leq d<|c|}|c|^{-2 s}$, where the sums are taken over all pairs $c, d$ of the ma$\operatorname{trix}\left(\begin{array}{l}* * \\ c d\end{array}\right) \subset \Gamma_{\infty} \backslash \Gamma / \Gamma_{\infty}$. Also the poles of the meromorphic function $S(s)$ are contained in the region $\Re s<1 / 2$ and in the interval $[1 / 2,1]$.

\section{Further analysis of the results}

To summarize, we propose that our results can be applied in the following problems. 
Finite volume cosets $\Gamma \backslash G / K$ and Killing spinors. In the previous sections we have discussed supergravity solutions involving anti de Sitter and real hyperbolic space factors. Hyperbolic spaces have infinite volume with respect to the Poincaré metric. Thus there are no normalizable modes for any field configurations in hyperbolic spaces. On the other hand, non-empty bulk and boundary field theories can be obtained by forming the coset spaces with topology $\Gamma \backslash \mathbb{H}^{N}$.

The hyperbolic manifolds $\mathbb{H}^{N}$, as factors in solution (i) of Sec. 2, admit Killing spinors. However, having the space forms (ii), (iii) of Sec. 2: $A d S_{3} \times \mathbb{H}^{2} \times \mathbb{H}^{2} \times \mathbb{S}^{4}, A d S_{2} \times \mathbb{H}^{2} \times \mathbb{H}^{3} \times \mathbb{S}^{4}$, as the solutions of supergravity theory, one can recognize that the factors $\mathbb{H}^{2} \times \mathbb{H}^{2}, \mathbb{H}^{2} \times \mathbb{H}^{3}$ cannot leave any unbroken supersymmetry. Indeed, the following result holds.

Proposition 1. (T. Friedrich [17]) A Riemannian spin manifold $\left(M^{N}, g\right)$ admitting a Killing spinor $\psi \neq 0$ with Killing number $\mu \neq 0$ is locally irreducible.

Proof. Let the locally Riemannian product be in the form $M^{N}=M^{K} \times M^{N-K}$. Let $\mathcal{X}, \mathcal{Y}$ be vectors tangent to $M^{K}$ and $M^{N-K}$ respectively, and, therefore, the curvature tensor of the Riemannian manifold $\left(M^{N}, g\right)$ is trivial. Since $\psi$ is a Killing spinor the following equations hold (see also Eq. (2.7)):

$$
\begin{aligned}
& \nabla_{X} \psi=\mu X \cdot \psi, \quad 4 \mu^{2}=[N(N-1)]^{-1} R \\
& \text { at each point of a connected Riemannian spin manifold }\left(M^{N}, g\right),
\end{aligned}
$$

where $R$ is a scalar curvature. Because of $(5.1)$ we have

$$
\begin{aligned}
& \nabla_{X} \nabla_{Y} \psi=\mu\left(\nabla_{X} \mathcal{Y}\right) \cdot \psi+\mu^{2} \mathcal{Y} \cdot x \cdot \psi \Longrightarrow \\
& \left(\nabla_{X} \nabla_{Y}-\nabla_{Y} \nabla_{X}-\nabla_{[X, Y]}\right) \psi=\mu^{2}(\mathcal{Y} \cdot x-x \cdot \mathcal{Y}) \psi .
\end{aligned}
$$

The curvature tensor $R(X, \mathcal{Y})$ in the spinor bundle $\mathfrak{S}$ is related to the curvature tensor of the Riemannian manifold $\left(M^{N}, g\right): R(X, \mathcal{Y})=(1 / 4) \sum_{j=1}^{N} e_{j} R(X, \mathcal{Y}) e_{j} \cdot \psi$, where $\left\{e_{j}\right\}_{j=1}^{N}$ is a orthogonal basis in the manifold. Therefore Eq. (5.2) can also be written as

$$
\sum_{j=1}^{N} e_{j} R(X, \mathcal{Y}) e_{j} \psi+[N(N-1)]^{-1} R(X \mathcal{Y}-\mathcal{Y} X) \psi=0
$$

From Eq. (5.3), we get $R \cdot X \cdot \mathcal{Y} \cdot \psi=0$, and moreover $X$ and $\mathcal{Y}$ are orthogonal vectors. Since $\mu \neq 0$ $(R \neq 0)$ it follows that $\psi=0$, hence a contradiction.

Simple type IIB supergravity backgrounds with D3 brane charge and constant dilaton are the following: $A d S_{3} \times \mathbb{H}^{2} \times \mathbb{S}^{5}, A d S_{2} \times \mathbb{H}^{3} \times \mathbb{S}^{5}$. Like $A d S_{5} \times \mathbb{S}^{2} \times \mathbb{S}^{3}$, these spaces have no Killing spinors and the solution is not supersymmetric [6]. But this solution is regular everywhere (being a direct product of Einstein spaces), and $\alpha^{\prime}$ corrections can be made very small for sufficiently large radius of compact part. Although non-supersymmetric, these spaces are an interesting setup for string compactification, in particular, for the construction of conformal field theories. Other compactifications can be obtained by replacing hyperbolic space factors $\mathbb{H}^{4}$ by any Einstein space $X^{4}$ of the same negative curvature, leading to the solution $A d S_{3} \times X^{4} \times \mathbb{S}^{4}$. In fact we have the following statement: 
Proposition 2. (T. Friedrich [17]) Let $\left(M^{N}, g\right)$ be a connected Riemannian spin manifold and let $\psi$ be a non-trivial Killing spinor with Killing number $\mu \neq 0$. Then $\left(M^{N}, g\right)$ is an Einstein space.

Proof. The proof easily follows from Proposition 2; indeed $\left(M^{N}, g\right)$ is an Einstein space of scalar curvature given by Eq. (5.1).

A relevant question is whether supersymmetry survives under the orbifolding by the discrete group $\Gamma$. This question could be addressed also to the factors $\Gamma \backslash\left(\mathbb{H}^{2} \times \mathbb{H}^{2}\right), \Gamma \backslash\left(\mathbb{H}^{2} \times \mathbb{H}^{3}\right)$. Perhaps there are more complicate solutions involving real hyperbolic spaces, where some supersymmetries are unbroken. However analysis of that problem is complicate and we leave it for another occasion. In fact, supersymmetry guarantees the stability of the physical system. But its absence does not necessarily implies instability. In general, a definite statement for the stability of supergravity solutions needs a study of the spectral properties of the operators, which are not well studied yet.

String-supergravity correspondence. A version of duality states that string theory (or $\mathrm{M}$ theory) compactified on spaces of the form $A d S_{D+1} \times\left(\Gamma \backslash \mathbb{H}^{N}\right) \times \mathbb{S}^{K}$ defines a $D$-dimensional conformal field theory with $S O(K+1)$ global symmetry. In addition, the correlation functions of the conformal field theory are defined as follows [18, 19] $\left\langle\exp \left\{\int d^{D} x \Phi_{\text {boundary }}(x) O(x)\right\}\right\rangle_{\mathrm{CFT}} \equiv$ $Z_{\text {string }}\left(\Phi_{\text {boundary }}(x)\right)$, where $Z_{\text {string }}$ is the partition function of the string theory computed with boundary values of the string fields, which act as sources of operators of conformal theory. In the classical limit of supergravity the string partition function can be evaluated as follows: $Z_{\text {string }} \cong$ $\exp \left[-I_{S G}(\Phi)\right]$, where the solution of the equations of motion in the background $A d S_{D+1} \times\left(\Gamma \backslash \mathbb{H}^{N}\right) \times$ $\mathbb{S}^{K}$ with the boundary condition $\Phi_{\text {boundary }}$, has to be taken into account.

Following the lines of [6] we mention here two interesting cases of supergravity solutions. First, the $D=11$ supergravity solution of the form $A d S_{5} \times M^{2} \times \mathbb{S}^{4}$. This model is dual to a $D=4$ non-supersymmetric conformal field theory with $S O(5)$ global symmetry group. The supergravity solution has M5-brane charge and one may expect the theory to be related to the six-dimensional $(2,0)$ or $(1,0)$ conformal field theories. The second case is the eleven-dimensional supergravity solution $A d S_{4} \times M^{3} \times \mathbb{S}^{4}$, and the type IIB solution $A d S_{3} \times M^{2} \times \mathbb{S}^{5}$. The $A d S_{4} \times M^{3} \times \mathbb{S}^{4}$ solution has M5 brane charge. Thus, the dual field theory is a $D=2+1$ conformal field theory associated with the $6 D(2,0)$ conformal theory, with an internal global symmetry group $S O(5)$. Finally the later solution has $\mathrm{D} 3$ brane charge and it should be dual to some $\mathcal{N}=0 D=1+1$ conformal field theory related to $\mathcal{N}=4 D=3+1$ super Yang-Mills theory.

Results on the holographic principle. The backgrounds considered in previous sections can be used for the construction of new $\mathcal{N}=0$ conformal field theories by holography. According to the holographic principle, there exist strong ties between certain field theories on a manifold ("bulk space") and on its boundary (at infinity). A few mathematically exact results relevant to that program are the following. The class of Euclidean $\mathrm{AdS}_{3}$ spaces which we have considered here are quotients of the real hyperbolic space $\mathbb{H}^{3}$ by a Schottky group. The boundaries of these spaces can be compact oriented surfaces with conformal structure (compact complex algebraic curves).

In [20], a principle associated with the Euclidean $\mathrm{AdS}_{2}$ holography has been established. The bulk space is there a modular curve, which is the global quotient of the hyperbolic plane $\mathbb{H}^{2}$ by 
a finite index group, $\Gamma$, of $G=P S L(2, \mathbb{Z})$. The boundary at infinity is then $P^{1}(\mathbb{R})$. Let $M$ be a coset space $M=\Gamma \backslash G$. Then, the modular curve $X_{\Gamma}:=\Gamma \backslash \mathbb{H}^{2}$ can be presented as the quotient $X_{\Gamma}=G \backslash\left(\mathbb{H}^{2} \times M\right)$; its non-commutative boundary (in the sense of Connes [21]) as the $C^{*}-$ algebra $C\left(P^{1}(\mathbb{R}) \times M\right)>\triangleleft G$, Morita equivalent to $C\left(P^{1}(\mathbb{R})\right)>\triangleleft \Gamma[22,20]$. The results which have been regarded as manifestations of the holography principle are [20]:

- There is a correspondence between the eigenfunctions of the transfer operator $L_{s}$ and the eigenfunctions of the Laplacian (Maas wave forms).

- The cohomology classes in $H_{1}\left(X_{\Gamma}\right.$, cusps, $\left.\mathbb{R}\right)$ can be regarded as elements in the cyclic cohomology of the algebra $C\left(P^{1}(\mathbb{R}) \times M\right)>\triangleleft G$. Cohomology classes of certain geodesics in the bulk space correspond to projectors in the algebra of observables on the boundary space.

- An explicit correspondence exists between a certain class of fields in the bulk space (Mellin transforms of modular forms of weight two) and the class of fields on the boundary.

Other constructions associated with the symmetric space can be considered for convex cocompact groups. In fact, let $\partial X$ be a geodesic boundary of the symmetric space $X$ of a real, rank one, semisimple Lie group $G$. If $\Gamma \subset G$ is a discrete torsion-free subgroup, then a $\Gamma$-equivalent decomposition, $\partial X=\Omega \cup \Lambda$, can be constructed, where $\Lambda$ is the limit set of $\Gamma$. The subgroup $\Gamma$ is called convex cocompact if $\Gamma \backslash X \cup \Omega$ is a compact manifold with boundary [23]. The geometry boundary of $\mathbb{H}^{N}$ in half-space Poincaré model is $\partial_{\infty} \mathbb{H}^{N}=\mathbb{R}^{N-1} \cup \infty$. If $\Gamma$ is convex cocompact and torsion free, then the orbit space, $X_{\Gamma}=\Gamma \backslash \mathbb{H}^{N}$, may be viewed as the interior of a compact manifold with boundary, namely the Klein manifold for $\Gamma, \bar{X}=\left(\Gamma \backslash \mathbb{H}^{N}\right) \cup(\Gamma \backslash \Omega(\Gamma))$, so that the boundary at infinity is given by $\partial_{\infty} X_{\Gamma}=\partial \bar{X}=\Gamma \backslash \Omega(\Gamma)$.

\section{Acknowledgements}

The authors would like to thank the Conselho Nacional de Desenvolvimento Científico e Tecnológico $(\mathrm{CNPq})$ for a support.

\section{References}

[1] M. J. Duff, B. E. Nilsson and C. N. Pope, Kaluza-Klein Supergravity, Phys. Rep. 130 (1986) 1.

[2] P. Breitenlohner and D. Z. Freedman, Stability in Gauged Extended Supergravity, Ann. Phys. (NY) 144 (1982) 249.

[3] Y. Fujii and K. Yamagishi, Killing Spinors on Spheres and Hyperbolic Manifolds, J. Math Phys. 27 (1986) 979.

[4] H. Lu, C. N. Pope and P. Townsend, Domain Walls from Anti-de Sitter Spacetime, Phys. Lett. B391 (1997) 39 [hep-th/9607164].

[5] H. Lu, C. N. Pope and J. Rahmfeld, A Construction of Killing Spinors on $S^{n}$, J. Math. Phys. 40 (1999) 4518 [hep-th/9805151].

[6] A. Kehagias and J. G. Russo, Hyperbolic Spaces in String and M-Theory, JHEP 0007 (2000) 027 [hep-th/0003281]. 
[7] S. Kachru and E. Silverstein, 4d Conformal Field Theories and Strings on Orbifolds, Phys. Rev. Lett. 80 (1998) 4855 [hep-th/9802183].

[8] A. Lawrence, N. Nekrasov and C. Vafa, On Conformal Theories in Four Dimensions, Nucl. Phys. B533 (1998) 199 [hep-th/9803015].

[9] S. Ferrara, A. Kehagias, H. Partouche and A. Zaffaroni, Membranes and Fivebranes with Lower Supersymmetry and their AdS Supergravity Duals, Phys. Lett. B431 (1998) 42 [hep-th/9803109].

[10] A. A. Tseytlin, Composite BPS Configurations of p-Branes in 10 and 11 Dimensions, Class. Quant. Grav. 14 (1997) 2085 [hep-th/9702163].

[11] H. Moscovici and R. J. Stanton, R-Torsion and Zeta Functions for Locally Symmetric Manifolds, Invent. Math. 105 (1991) 185.

[12] J. Park, Eta Invariants and Regularized Determinants for Odd Dimensional Hyperbolic Manifolds with Cusps, math.DG/0111175.

[13] D. Barbasch and H. Moscovici, $L^{2}$-Index and the Selberg Trace Formula, J. Funct. Anal. 53 (1981) 151.

[14] E. Elizalde, S. D. Odintsov, A. Romeo, A. A. Bytsenko and S. Zerbini, Zeta Regularization with Applications, World Scientific, Singapore, 1994.

[15] A. A. Bytsenko, G. Cognola, L. Vanzo and S. Zerbini, Quantum Fields and Extended Objects in Space-Times with Constant Curvature Spatial Section, Phys. Rep. 266 (1996) 1.

[16] A. A. Bytsenko, G. Cognola, E. Elizalde, V. Moretti and S. Zerbini, Analytic Aspects of Quantum Fields, World Scientific, Singapore, 2003.

[17] T. Friedrich, Dirac Operators in Riemannian Geometry, Graduate Studies in Mathematics, vol. 25, AMS, Providence 1997.

[18] S. S. Gubser, I. R. Klebanov and A. M. Polyakov, Gauge Theory Correlators from Non-Critical String Theory, Phys. Lett. B428 (1998) 105 [hep-th/9802109].

[19] E. Witten, Anti De Sitter Space And Holography, Adv. Theor. Math. Phys. 2 (1998) 253 [hep-th/9802150].

[20] Yu. I. Manin and M. Marcolli, Holography Principle and Arithmetic of Algebraic Curves, Adv. Theor. Math. Phys. 5 (2002) 617 [hep-th/0201036].

[21] A. Connes, Non-Commutative Geometry, Academic Press, New York, 1994.

[22] M. Marcolli, Limiting Modular Symbols and the Lyapunov Spectrum, math.NT/0111093.

[23] S. J. Patterson and P. A. Perry, The Divisor of Selberg's Zeta Function for Kleinian Groups, Duke Math. J. 106 (2001) 321. 